\title{
Associations of isokinetic knee steadiness with hop performance in patients with ACL deficiency
}

\author{
Yong-Hao Pua ${ }^{1}$, Peck-Hoon Ong ${ }^{1}$, Jia-Ying $\mathrm{Ho}^{1}$, \\ Adam L. Bryant ${ }^{2}$, Kate Webster ${ }^{3}$, Ross A. Clark ${ }^{4}$
}

1. Department of Physiotherapy, Singapore General Hospital, Singapore

2. Department of Physiotherapy, The University of Melbourne, Melbourne, Australia

3. School of Health Sciences Research, La Trobe University

4. School of Exercise Science, Australian Catholic University

Proposed Journal: Knee Surgery, Sports Traumatology, Arthroscopy

Running Head: Knee torque steadiness in ACLD

\author{
Corresponding Author \\ Yong-Hao Pua PhD \\ Department of Physiotherapy \\ Singapore General Hospital \\ Outram Road \\ Singapore 169608 \\ Email: puayonghao@gmail.com \\ Fax: +6563215495 \\ Tel: +656321 4132
}

\section{ABSTRACT}

Purpose: Contrary to the ample data available regarding the functional significance of isokinetic knee strength in patients with anterior cruciate ligament deficiency (ACLD), much less is known about the functional significance of isokinetic knee steadiness. This cross-sectional study aimed to evaluate, in patients with ACLD, the independent impact of isokinetic quadriceps and hamstrings torque steadiness on single-leg hop performance.

Methods: Eighty-seven patients with unilateral ACLD participated. Patients performed isokinetic quadriceps and hamstrings steadiness and strength testing at $60 \% \mathrm{sec}$ on an isokinetic dynamometer. Muscle steadiness and 
strength were represented by the wavelet-derived mean instantaneous frequency and peak value of the torquetime curves, respectively. To measure hop performance, patients performed a single-leg hop for distance and a 6-metre single-leg hop for velocity.

Results: One of two patients $(\mathrm{n}=45[51 \%])$ had a $10 \%$ or greater difference in knee torque frequency levels between the ACLD and contralateral knees. In multivariable models adjusted for age, sex, knee pain, and knee strength, hamstrings steadiness was significantly related with hop velocity whilst quadriceps steadiness was significantly related with both hop distance and velocity. Variance decomposition analyses suggested that quadriceps steadiness was similar in importance to hamstrings strength on hop distance and velocity.

Conclusions: In patients with ACLD, isokinetic knee steadiness deficits were common and were independently associated with single-leg hop performance. Knee torque steadiness - a heretofore understudied variable - may prove a useful adjunct to conventional peak torque measurements by offering additional information to researchers and rehabilitation professionals about muscle performance and neuromuscular knee control.

Level of Evidence: Prognostic Studies: Level III

Key Terms: Force control; Isokinetics; Knee; ACL deficiency; Single leg hop test 


\section{INTRODUCTION}

In patients with an anterior cruciate ligament deficiency (ACLD), isokinetic knee testing is routinely performed in research and in clinical practice to gather information on knee muscle performance[37]. In an isokinetic knee test, knee muscle strength - that is, the highest value of the torque-time curve - represents the quantity of muscle torque output. On the other hand, knee muscle steadiness - that is, the smoothness of the torque-time curve - represents the quality of muscle torque output. Computationally, knee steadiness is the mean frequency of the small but rapid torque fluctuations in a torque-time curve, over and above the natural isokinetic movement frequency[9,11]. Accordingly, lower frequency values indicate greater knee torque steadiness and greater smoothness of the torque-time curve[42].

Physiologically, the smoothness of torque generation reflects motor output variability[17,30] which, in turn, influences the precision of voluntary movements[13]. Given that torque steadiness also depends on adequate joint stability and muscle coordination[3,41,43], it seems plausible that knee steadiness is an important predictor of physical function in patients with ACLD because it provides information about neuromuscular control. Supporting this, we and others have reported lower hamstrings and quadriceps torque steadiness in patients with ACLD than in knee-healthy controls[9,11,43]. More important, we have shown that knee torque steadiness was associated with single-leg hop performance[9,11]. Not much has been written since which further illuminates the functional significance of these knee steadiness variables; however, our previous work was limited on three counts. First, our previous work examined patients with ACLD who did not require an ACL reconstruction ("copers") which, in turn, limited the generalizability of our results to more heterogeneous populations - for example, patients who are awaiting an ACL reconstruction. Second, we studied only 13 patients with ACLD and hence, we do not know whether the predictive power of the knee steadiness variables for hop performance adds to that of isokinetic knee strength. For knee steadiness to merit serious consideration as a stratification or outcome measure in both research and clinical settings, we believe it critical to examine its incremental and independent contribution to prediction beyond that of conventional isokinetic strength (peak torque) measures. Third, due also to a small sample size, we did not - and could not - simultaneously analyze the relative importance of quadriceps and hamstrings strength and torque steadiness in predicting hop performance. Ostensibly, results from such analyses could provide direction on the isokinetic variables to focus on in both clinical and research settings. And until future studies have addressed the aforementioned concerns, recommendations to consider and measure knee steadiness in patients with ACLD cannot be fully justified. 
Thus, to provide stronger justifications for the potential clinical and research use of the knee steadiness measures, the purpose of this study was to, in a large sample of patients with ACLD, examine the independent impact and relative importance of quadriceps and hamstrings torque steadiness measures on two wellestablished tests of dynamic knee stability[18,24] - namely, the single-leg hop test for distance and the 6-metre timed hop test. It was hypothesized that (i) quadriceps and hamstrings steadiness measures would be significantly related with both hop distance and velocity, and that (ii) knee steadiness measures would be as important as the knee strength measures in influencing hop performance.

\section{MATERIALS AND METHODS}

Our study sample comprised patients with ACLD undergoing unilateral ACL reconstruction at a large tertiary institution in Singapore, from November 2011 to April 2013. ACL rupture was confirmed by diagnostic magnetic resonance imaging and by manual laxity testing using a KT-2000 arthrometer (MEDmetric, San Diego, California). Patients were recruited within a month before their surgery as part of an ongoing cohort study investigating the preoperative predictors of postoperative physical activity levels. The present study is concerned with the preoperative data. Patients were excluded if they (i) had previous or concurrent posterior cruciate ligament injury, (ii) had knee extension range-of-motion deficits greater than $10^{\circ}$, (iii) had significant neck, back or other non-knee pain, or (iv) had any medical conditions that would compromise physical function or affect their ability to complete testing. A total of 103 eligible patients participated in this study. As a safety precaution, we excluded from the single-leg hop tests 8 patients whose body-weight adjusted isokinetic quadriceps strength (described later) was below $1.0 \mathrm{Nm} / \mathrm{kg}$. Additionally, 8 patients were unable or unwilling to perform consecutive, single-leg hops; hence, we included patients in this study for whom hop performance data were complete. The final sample (87 of 103) comprised patients (mean[SD] age, 26[6] years) who were predominantly male $(85 \%)$, and the median time from injury to study enrolment was 56 days (interquartile range, 21 to 168). Of these 87 patients, $34(39 \%)$ had concomitant meniscal injury and $76(87 \%)$ participated in competitive sports (Tegner activity level 7 and above) before ACL rupture. Overall, the mean self-report functional level, indexed by the Lower Extremity Functional Scale [6], was 61 points (SD, 9.4) out of a possible 80.

All patients attended the baseline session at the outpatient physiotherapy department after providing written informed consent. Prior to the physical assessment, patients warmed up by cycling for 10 minutes on a 
stationary bicycle. Testing was performed in the following order: isokinetic knee test, hop for distance test, and 6-metre hop test. Of note, the order of the physical measures was not randomized and the least stressful test was placed before the most stressful test to reduce the potential confounding influence of pain. Patients were also given rest breaks between tests to minimize fatigue. After each test, patients rated their knee pain intensity on an 11-point visual numeric pain scale, with 0 indicating 'no pain' and 10 indicating 'worst pain ever experienced.'

\section{Testing}

\section{Isokinetic Testing}

To measure knee muscle performance, maximal-effort concentric contractions of the quadriceps and hamstrings were obtained on a Biodex Medical IV isokinetic dynamometer (Shirley, NY, USA). The patient was tested in a seated position with the hip at $90^{\circ}$ of flexion, and strapping was placed across the waist and chest to stabilize the torso. The axis of rotation of the dynamometer lever arm was aligned to the femoral lateral condyle whilst the lever arm was secured to the tibia just proximal to the medial malleolus via an ankle cuff. Before testing the knee muscles, the gravity compensation procedure was performed by measuring the patient's passive limb weight at $30^{\circ}$ of knee flexion. Following a warm-up comprising 3 submaximal and 2 maximal contractions, all patients performed 5 consecutive maximal-effort extension and flexion repetitions. To facilitate maximum performance, patients were instructed to extend and flex their knee as fast and forcefully as possible, and strong verbal encouragement was given during testing. To minimize the contribution of unwanted muscles, patients maintained a crossed-arms position during testing. To allow patients to gain confidence with the testing protocol, we tested the contralateral healthy knee before the ACLD knee. Our isokinetic protocol used an angular velocity of $60^{\circ} \mathrm{s}$ and the range-of-motion was set from $90^{\circ}$ to $40^{\circ}$ of knee flexion. We chose this testing range-of-motion because our patients would be retested 3 months post ACL reconstruction, and testing in the last $30^{\circ}$ of knee extension at that time would be contraindicated as terminal knee extension was reportedly associated with substantial shear forces transmitted to the ACL graft[44]. Thus, to allow valid comparison within patients and between sessions, our testing range-of-motion specifically avoided this range. Relatedly, because our testing range-of-motion was relatively narrow, we chose a low preset angular velocity $(60 \%)$ to maximize the range-of-motion within which the limb was moving at a constant (preset) velocity[31]. 


\section{Single-leg Hop for Distance Test}

The single-leg hop for distance test was previously described by Daniel et al[15]. For this test, patients stood on one leg, hopped, and landed on the same limb. Patients were instructed to hop maximally for distance and to maintain their balance on landing for at least 2 seconds. Each patient performed two submaximal practice trials followed by three test trials. Additional trials were provided if patients reported a failure to achieve maximum effort. To allow patients to gain confidence with the testing protocol, all tests were performed on the uninjured side followed by the ACLD side. From a measuring tape that was affixed to the floor, the distance hopped was measured at the level of the great toe and recorded to the nearest millimeter. In this study, for each patient, the highest measurement of all valid trials performed on the ACLD side was analyzed. One recent cohort study of 91 non-operatively treated patients with ACLD found that the single-leg hop for distance test predicted future (1-year) levels of self-reported knee function (indexed by the International Knee Documentation Committee Subjective Knee Form)[20].

\section{Six-metre Hop Test}

The 6-metre hop test was previously described by Barber et al[4]. For this test, patients stood on the ACLD limb and hopped an 8-m walkway. Patients were instructed to hop as quickly as they could without compromising safety. To remove the subjective errors associated with the use of handheld stopwatches, a dualbeam timing gate system (Nintendo Wii hand controllers)[14] was used to measure, to the nearest millisecond, the time taken for the patient to cover the first 6metres. No practice trial was given and each patient performed two trials, of which the faster trial was used to compute hop velocity (i.e., 6metres/hop time). Here, we should mention that although the 6-metre hop performance is traditionally analyzed in the time domain[4], we chose to express it in velocity units for 2 reasons. First, our time data were positively skewed and its distribution was satisfactorily normalized with a reciprocal - but not logarithmic - transformation. Second, the concept of hop velocity is clinically meaningful and easily understood by clinicians, and the calculation of velocity basically involves a reciprocal transformation of time (fixed distance/time). Fitzgerald et al[18] have shown, in patients with ACLD, that a combination of the 6-m timed hop test and other clinical measures could discriminate those with poor dynamic knee stability from those without it. 


\section{Data Analysis}

To ensure that our knee strength and steadiness variables were computed from torque data generated when the test limb was moving at a constant (preset) velocity[26], we extracted isokinetic torque data recorded between $85^{\circ}$ and $45^{\circ}$ of knee flexion. To eliminate the gravitational effects of limb mass on the torque data, the passive limb weight was added to and subtracted from the quadriceps and hamstrings torque values, respectively. Among the 5 maximal-effort repetitions produced by the patient, quadriceps and hamstring strength represented the highest torque achieved by the respective muscle groups and these peak torque values (measured to the nearest $0.1 \mathrm{Nm}$ ) were normalized to the patient's body weight (i.e., peak torque $[\mathrm{Nm}] /$ body weight $[\mathrm{kg}]$ )[27].

The steadiness of the quadriceps and hamstrings torque signal was derived using the analytic wavelet transform technique described in our previous work[9,11]. Briefly, this consisted in using the complex Morlet wavelet to convert the isokinetic signal into multiple time-scales, then calculating the mean instantaneous frequency (MIF) at each sampling point of the signal. The average MIF within the region of interest - that is, between $85^{\circ}$ and $45^{\circ}$ of knee flexion - was calculated for each of the 5 trial repetitions, with the median MIF (measured to the nearest $0.1 \mathrm{~Hz}$ ) used to represent knee torque steadiness. Noteworthy, a difference in analysis between our previous $[9,11]$ and present work is the detrending of the signal prior to calculation of MIF in the present study, leaving only the higher frequency oscillations. Specifically, this consisted in performing $1.6 \mathrm{~Hz}$ to $12.5 \mathrm{~Hz}$ bandpass filtering of the torque data, implemented using a Daubechies 4 discrete wavelet transform with the appropriate approximation and detail levels removed. This method of detrending was chosen because our preliminary analysis revealed it to be adaptive to different fundamental patterns in the signal (for example, mild skewness) whilst allowing for extraction of signal oscillations which are associated with unsteady movement. Put otherwise, the present analysis scheme is chosen because it amplifies the higher frequency oscillations that we believe are representative of unsteady torque production[42]. Figure 1 provides an example of the original, extracted low-frequency, and retained high-frequency torque signal for a knee extension (quadriceps) test repetition.

This study was approved by the SingHealth Centralized Institutional Review Board (IRB Ref No: 2011/387/D) and was conducted in accordance with the Declaration of Helsinki. 


\section{Statistical analysis}

Sample size calculation was based on the guideline of at least 15 patients per degree of freedom (i.e., per regression coefficient estimated) [23], which resulted in an estimated sample size of 75 patients for five degrees of freedom in a multivariable regression model. We used descriptive statistics to characterize the study sample: we used means with SDs and medians with interquartile ranges for continuous variables and frequencies with percentages for categorical variables. We used a paired $t$-test to compare the ACLD and contralateral healthy knees on the torque steadiness levels. For descriptive purposes, we also calculated the proportion of patients (with Wilson 95\% confidence intervals[35]) whose isokinetic torque frequency levels were $10 \%$ or greater (poorer steadiness) in the ACLD knee than in the contralateral knee.

To examine and to visualize the interrelations between isokinetic knee variables and hop performance, we used a scatterplot matrix with Pearson's product-moment correlation coefficients. To examine the independent associations of the isokinetic quadriceps and hamstrings variables with hopping performance, we used separate multivariable regression models and included in each model three a priori defined covariables - namely, age, sex, and knee pain. To minimize model overfitting, we used principal component analysis to combine four knee pain measures (two knee pain measures from isokinetic testing and two measures from hop testing) into a single (first) component variable ("knee pain"). To facilitate clinical interpretation of the regression output, we calculated inter-quartile range regression coefficients for all continuous variables [23].

To evaluate the relative importance of the various isokinetic knee variables in predicting hop performance, we regressed hop performance on all isokinetic knee variables and used the variance decomposition technique developed by Lindeman, Merenda, and Gold (LMG)[32]. Specifically, the LMG technique calculates the model $R^{2}$ increment from adding a given knee variable to a model without it and averaging the $R^{2}$ increments from all permutations of variable entry. Accordingly, this "averaging over orderings" technique produces, for each knee variable, a metric that quantifies the proportionate contribution the variable makes to the prediction of hop performance by itself and in combination with other knee variables[22,29].

Statistical analyses were done with R version 2.15 .2 (http://www.r-project.org), and the LMG technique was performed with the relaimpo R package[21]. Statistical significance was determined at the 2-sided 0.05 level. 


\section{RESULTS}

Table 1 shows the patients' characteristics. In this sample, the mean pain score during isokinetic and hop testing was below 2 points, indicating a generally low degree of pain. The distributions of quadriceps and hamstrings torque steadiness (torque frequency) of the ACLD knees were approximately normal with means of 2.53 (SD, $0.65)$ and 2.28 (SD, 0.46), respectively. Quadriceps and hamstrings steadiness (torque frequency) levels were $18 \%$ (mean absolute difference, $0.38 \mathrm{~Hz} ; P<0.001)$ and $10 \%(0.20 \mathrm{~Hz} ; P<0.001)$, respectively, higher in the ACLD knees than in the contralateral knees. Among our sample of 87 patients, just over half ( $n=45$ [51\%]; $95 \%$ CI, $40 \%$ to $61 \%$ ) had a $10 \%$ or greater side-to-side difference in either quadriceps or hamstrings torque frequency.

The scatterplot matrix in Figure 2 demonstrates the interrelations between the isokinetic knee variables and hop performance. Quadriceps and hamstrings strength correlated with hop performance ( $r$-values, 0.51 to 0.64 , $P$ 's $<0.001)$. Significant correlations were also present for both quadriceps steadiness $(r=-0.46, P<0.001$ for both hop distance and hop velocity) and hamstrings steadiness (hop distance, $r=-0.27, P=0.013$; hop velocity, $r=-$ $0.36, P<0.001)$.

Tables 2 and 3 show the multivariable models for the single-leg hop distance and 6-metre hop velocity, respectively. The combination of isokinetic knee variables and covariables explained between $25 \%$ and $48 \%$ of the adjusted variation in hop distance and velocity. Across the models, quadriceps and hamstrings strength were statistically significant predictors. In regard to the knee steadiness variables, hamstrings steadiness was significantly related only to hop velocity. For quadriceps steadiness, higher torque frequency values (poorer steadiness) were associated with shorter hop distance and lower hop velocity. Specifically, an increase in isokinetic quadriceps torque frequency of $0.76 \mathrm{~Hz}$ (equivalent to its interquartile range) was associated with $12.7 \mathrm{~cm}(95 \% \mathrm{CI}, 5.9$ to $19.4 \mathrm{~cm} ; P<0.001)$ shorter hop distance and $0.24 \mathrm{~m} / \mathrm{s}(95 \% \mathrm{CI}, 0.09$ to $0.38 \mathrm{~m} / \mathrm{s} ; P<0.01)$ lower hop velocity, respectively. Noteworthy, the observed effect size for hop distance $(12.7 \mathrm{~cm})$ was comparable to the minimal detectable change scores reported in knee-healthy individuals[38] $(10.7 \mathrm{~cm})$ and in patients with an ACL reconstruction[7] $(12.0 \mathrm{~cm})$.

Figure 3 shows the relative importance of the various isokinetic knee variables in predicting hop distance $\left(R^{2}=0.45, P<0.001\right)$ and hop velocity $\left(R^{2}=0.49, P<0.001\right)$. Based on the LMG metric, quadriceps strength was 
the most influential variable, accounting for $\sim 22 \%$ of the variance in hop distance and velocity. Quadriceps steadiness was similar in importance to hamstrings strength on hop distance and velocity, with each variable accounting for $\sim 10 \%$ of the variance. Hamstrings steadiness explained the least amount of variance in hop performance $(<6 \%)$.

\section{DISCUSSION}

The present study, involving 87 patients with ACLD, has 3 important findings. First, torque frequency levels of the quadriceps and hamstrings were higher in the involved than in the contralateral knees. Second, greater knee torque steadiness (lower torque frequency levels) was associated with greater single-leg hop distance and velocity, independent of conventional isokinetic knee strength, age, sex, and knee pain. Third, the relative importance analyses indicated that the influence of quadriceps steadiness on hop performance was similar in importance to that of hamstrings strength. Overall, these findings support our hypothesis that isokinetic knee steadiness measures wielded an independent and important influence on hop performance.

Despite some differences in isokinetic testing protocols and data-processing techniques, our current and previous[9,11] results reinforced those of the paper by Tsepis et al[43] that quantified and reported lower quadriceps and hamstrings torque steadiness (higher torque frequency levels) in the ACLD than in the contralateral knees. But unlike previous authors, we further sought to determine the association of knee steadiness with hop performance. In our previous work on a mixed sample of 38 patients with ACLD (n=13) and ACL reconstruction ( $n=25)$, we observed that greater quadriceps steadiness (lower torque frequency) was related to longer time to complete a timed-hop test (poorer performance)[11]. In our current study, quadriceps steadiness was associated with hop performance, but in the opposite direction - that is, greater quadriceps steadiness was associated with better hop performance. On the other hand, however, we observed that greater hamstrings steadiness was associated with greater hop velocity, and this finding was generally in accordance with those of our prior work[9]. The reasons for these discrepancies are uncertain but we should mention that the present study (i) was over 2 -fold larger than our previous study ( $n=87$ vs 38 ), (ii) focused specifically on patients with ACLD ( $n=87$ vs 13), and (iii) used multivariable analyses which, in combination, allowed us to draw more robust conclusions. Equally important, our prior study examined a group of "copers" whereas all patients in the present study were awaiting an ACL reconstruction and their single-leg hop performance varied 
substantially (Table 1). Ostensibly, potential differences in neuromuscular strategies between "copers" and “non-copers”[18] may preclude a direct comparison of results between our previous and present work.

Nevertheless, what plausible mechanisms might link reduced knee steadiness - in particular, reduced quadriceps torque steadiness - to poor hop performance in our present study? One speculation is that because maximaleffort knee extension movements during isokinetic testing can potentially induce anterior tibial translation and mechanical instability in the ACL deficient knee[44], an adaptive neuromuscular strategy by patients may consist in increased activation of the hamstrings to produce posterior tibial translation to compensate for joint laxity $[1,2,10,11,44]$ which, in turn, facilitates muscle coordination and steady torque generation[43]. Equally, adequate hamstrings co-activation and torque generation may be a beneficial strategy used by more functional patients with ACLD to increase dynamic knee stability and achieve better hop performance[10]. In contrast, poorly-regulated and inadequate hamstrings coactivation or moment output allows excessive anterior tibial shearing, reduces dynamic knee stability, and impairs quadriceps torque steadiness and hop performance[39,43]. Supporting this notion, greater hamstrings strength was related to lower quadriceps torque frequency levels (greater steadiness) in the present study $(r=-0.31, P<0.001$; Figure 2$)$ whilst some indirect evidence also exists to suggest an association between generalized joint laxity and reduced knee torque steadiness[28]. Nevertheless, we acknowledge that the literature on the association between muscle (antagonist) coactivation and torque steadiness is limited and mixed[3,9,11,12,16,28,41], and that we did not have EMG data in this present study, which would have allowed for a more definitive interpretation of our results. Beside the level of hamstrings coactivation or torque generation, other physiological factors - such as variability in the discharge behaviour of recruited motor units within the agonist musculature (that is, the quadriceps) - may also potentially influence the smoothness of torque production[40,45]. Indeed, it is possible for factors that exaggerate size differences among active motor units - for example, reflex-mediated atrophy of Type II muscle fibers that can occur as a result of knee pain or effusion in the months following an ACL injury[5,33,34] - to magnify torque fluctuations by increasing the relative contribution of individual motor units to the net force[12]. Regardless of the eventual explanation, as the first step, the link between quadriceps torque steadiness and hop performance has been established in our study comprising a relatively large sample of patients with ACLD, and future studies should explore its underlying mechanistic bases. 
Given that isokinetic knee testing for patients with ACLD is common in clinical and research settings, a natural question asks, "Which of the knee steadiness and strength measures should be preferred?" No direct evidence answers this critical question and our relative importance analysis is novel in demonstrating that while knee strength had a stronger association with hop performance than did knee steadiness, the contribution of knee steadiness - in particular, quadriceps steadiness - to hop performance was not marginal (Figure 3). These findings are biologically plausible and suggest that while knee strength is a prerequisite for maximal-effort functional activities, knee steadiness - an index of knee control and movement precision - is an important cofactor for the development of optimal functional performance. Although preliminary, our findings suggest that the predominant focus on isokinetic knee strength offers only an incomplete perspective on muscle performance. Indeed, given that knee strength correlated weakly to moderately with steadiness (Figure 3), our findings substantiate the literature[24,39] and the clinically based intuition that not all patients with adequate strength levels have adequate and adaptive neuromuscular control. For this reason, if our results were replicated, future research should expand our view beyond traditional strength measures by exploring whether patients with ACLD could be disaggregated into meaningful subgroups according to both isokinetic strength and steadiness levels.

The present study has implications other than those discussed earlier. First, at the broadest level, it is quite clear that the combination of isokinetic knee variables contributes substantially to hop performance which, in turn, counters previous nihilism about the "functionality" of open-chain, isokinetic knee testing in patients with ACLD[37]. Second, from a mechanistic viewpoint, our findings indicate that knee steadiness deficits are common and functionally relevant in ACLD; hence, understanding the neuromuscular dysfunction that underlies these deficits may potentially lead to refinement of theoretical models and development of rehabilitative strategies. Third, besides serving as a possible model system for understanding neuromuscular knee control, our measure of knee steadiness - derived from standard isokinetic knee data - might also be considered as an important, complementary, and convenient outcome measure in research studies and clinical practice involving isokinetic knee testing in patients with ACLD.

The present study has limitations. First, although our analyses were guided by theory and prior data, we acknowledge that causal conclusions are not possible due to the cross-sectional nature of our study; hence, our findings need to be replicated in independent populations using a longitudinal or intervention design. Second, 
our study comprised predominantly men which, in turn, limited the generalizability of our results to women.

That said, however, given what is known about the possible differences in torque steadiness, neuromuscular knee control, and hop performance between sex[8,25], it is possible that in a sample comprising a larger proportion of women, the effects of isokinetic knee steadiness on hop performance might be even more profound. Third, we measured only hop performance and it would be informative to evaluate, in a longitudinal fashion, the associations of isokinetic knee steadiness with other important clinical outcomes and consequences associated with an ACLD[19,36] - for example, knee re-injury rates and articular cartilage volume.

\section{Conclusion}

In conclusion, in patients with ACLD, isokinetic knee steadiness deficits were common and were independently associated with single-leg hop performance after adjustment for knee muscle strength and standard covariables. Furthermore, the influence of quadriceps steadiness on hop performance was similar in importance to that of hamstrings strength. These findings contribute new information to our understanding of knee muscle performance and control in ACLD and suggest that in both clinical and research settings, knee torque steadiness may prove a useful adjunct to conventional isokinetic strength measurements.

\section{FIGURE LEGENDS}

Figure $1 \quad$ Example data for a quadriceps test repetition with the original (raw), low frequency, and high frequency signal plotted. The mean instantaneous frequency (MIF) analysis performed in this study was performed only on the high frequency data. Consequently, the MIF value represents the mean frequency of the fluctuations occurring at speeds greater than the underlying fundamental frequency of the movement, which is controlled by the fixed velocity of the test movement.

Figure 2 Scatterplot matrix of isokinetic knee measures, hop distance, and 6-metre hop velocity. Diagonal panels show the kernel density plots of the variables. Lower diagonal panels show the scatterplots with loess smoothers and shaded regions are $95 \%$ CIs for the regression estimates. Upper diagonal panels show the Pearson's coefficients with $P$-values.

Figure 3 Relative importance of isokinetic quadriceps and hamstrings variables for predicting hop distance (left panel) and 6-metre hop velocity (right panel) based on the Lindeman, Merenda, and Gold (LMG) metric. Error bars are $95 \%$ bootstrap confidence intervals.

\section{ACKNOWLEDGEMENTS}

This project is grant funded by Singhealth Foundation start-up grant (SHF/FG476S/2010). We thank Tan Bee Yee, the head of the Department of Physiotherapy, Singapore General Hospital, for supporting this study; John Tan Wei-Ming for his assistance; Luke Perraton for his advice; and the orthopedic surgeons from the Singapore General Hospital for allowing us access to their patients. 


\section{REFERENCES}

1. Aagaard P, Simonsen EB, Andersen JL, Magnusson SP, Bojsen-Moller F, Dyhre-Poulsen P (2000) Antagonist muscle coactivation during isokinetic knee extension. Scand J Med Sci Sports 10 (2):58-67.

2. Alkjaer T, Simonsen EB, Magnusson SP, Dyhre-Poulsen P, Aagaard P (2012) Antagonist muscle moment is increased in ACL deficient subjects during maximal dynamic knee extension. Knee 19 (5):633-639.

3. Bandholm T, Rose MH, Slok R, Sonne-Holm S, Jensen BR (2009) Ankle torque steadiness is related to muscle activation variability and coactivation in children with cerebral palsy. Muscle Nerve 40 (3):402-410.

4. Barber SD, Noyes FR, Mangine RE, McCloskey JW, Hartman W (1990) Quantitative assessment of functional limitations in normal and anterior cruciate ligament-deficient knees. Clin Orthop Relat Res 255:204-214.

5. Baugher WH, Warren RF, Marshall JL, Joseph A (1984) Quadriceps atrophy in the anterior cruciate insufficient knee. Am J Sports Med 12 (3):192-195.

6. Binkley JM, Stratford PW, Lott SA, Riddle DL (1999) The Lower Extremity Functional Scale (LEFS): Scale development, measurement properties, and clinical application. Phys Ther 79 (4):371-383.

7. Brosky JA, Jr., Nitz AJ, Malone TR, Caborn DN, Rayens MK (1999) Intrarater reliability of selected clinical outcome measures following anterior cruciate ligament reconstruction. J Orthop Sports Phys Ther 29 (1):39-48.

8. Brown RE, Edwards DL, Jakobi JM (2010) Sex differences in force steadiness in three positions of the forearm. Eur J Appl Physiol 110 (6):1251-1257.

9. Bryant AL, Clark RA, Pua YH (2011) Morphology of hamstring torque-time curves following ACL injury and reconstruction: mechanisms and implications. J Orthop Res 29 (6):907-914.

10. Bryant AL, Creaby MW, Newton RU, Steele JR (2008) Dynamic restraint capacity of the hamstring muscles has important functional implications after anterior cruciate ligament injury and anterior cruciate ligament reconstruction. Arch Phys Med Rehabil 89 (12):2324-2331.

11. Bryant AL, Pua YH, Clark RA (2009) Morphology of knee extension torque-time curves following anterior cruciate ligament injury and reconstruction. J Bone Joint Surg Am 91 (6):1424-1431.

12. Burnett RA, Laidlaw DH, Enoka RM (2000) Coactivation of the antagonist muscle does not covary with steadiness in old adults. J Appl Physiol 89 (1):61-71.

13. Christou EA, Carlton LG (2001) Old adults exhibit greater motor output variability than young adults only during rapid discrete isometric contractions. J Gerontol A Biol Sci Med Sci 56 (12):B524-532.

14. Clark RA, Paterson K, Ritchie C, Blundell S, Bryant AL (2011) Design and validation of a portable, inexpensive and multi-beam timing light system using the Nintendo Wii hand controllers. J Sci Med Sport 14 (2):177-182.

15. Daniel DM, Stone ML, Riehl B, Moore MR (1988) A measurement of lower limb function: The one-leg hop for distance. Am J Knee Surg 1 (4):212-214.

16. Danion F, Gallea C (2004) The relation between force magnitude, force steadiness, and muscle cocontraction in the thumb during precision grip. Neurosci Lett 368 (2):176-180.

17. Enoka RM, Christou EA, Hunter SK, Kornatz KW, Semmler JG, Taylor AM, Tracy BL (2003) Mechanisms that contribute to differences in motor performance between young and old adults. J Electromyogr Kinesiol 13 (1):1-12. 
18. Fitzgerald GK, Axe MJ, Snyder-Mackler L (2000) A decision-making scheme for returning patients to highlevel activity with nonoperative treatment after anterior cruciate ligament rupture. Knee Surg Sports Traumatol Arthrosc 8 (2):76-82.

19. Frobell RB (2011) Change in cartilage thickness, posttraumatic bone marrow lesions, and joint fluid volumes after acute ACL disruption: a two-year prospective MRI study of sixty-one subjects. J Bone Joint Surg Am 93 (12):1096-1103.

20. Grindem H, Logerstedt D, Eitzen I, Moksnes H, Axe MJ, Snyder-Mackler L, Engebretsen L, Risberg MA (2011) Single-legged hop tests as predictors of self-reported knee function in nonoperatively treated individuals with anterior cruciate ligament injury. Am J Sports Med 39 (11):2347-2354.

21. Gromping U (2006) Relative importance for linear regression in R: the package relaimpo. J Stat Softw 17 (1):1-27.

22. Gromping U (2007) Estimators of relative importance in linear regression based on variance decomposition. Am Stat 61 (2):139-147.

23. Harrell Jr FE (2001) Regression modeling strategies: with applications to linear models, logistic regression, and survival analysis. Springer, New York.

24. Hurd WJ, Axe MJ, Snyder-Mackler L (2008) A 10-year prospective trial of a patient management algorithm and screening examination for highly active individuals with anterior cruciate ligament injury: Part 2, determinants of dynamic knee stability. Am J Sports Med 36 (1):48-56.

25. Hurd WJ, Axe MJ, Snyder-Mackler L (2008) Influence of age, gender, and injury mechanism on the development of dynamic knee stability after acute ACL rupture. J Orthop Sports Phys Ther 38 (2):3641 .

26. Iossifidou AN, Baltzopoulos V (1998) Inertial effects on the assessment of performance in isokinetic dynamometry. Int J Sports Med 19 (8):567-573.

27. Jaric S, Radosavljevic-Jaric S, Johansson H (2002) Muscle force and muscle torque in humans require different methods when adjusting for differences in body size. Eur J Appl Physiol 87 (3):304-307.

28. Jensen BR, Olesen AT, Pedersen MT, Kristensen J, Jens H, Remvig L, Simonsen EB, Juul-Kristensen B (2013) The effect of generalized joint hypermobility on knee function and muscle activation in children and adults. Muscle \& Nerve 48 (5):762-769.

29. Johnson JW, LeBreton JM (2004) History and use of relative importance indices in organizational research. Organ Res Meth 7 (3):238-257.

30. Jones KE, Hamilton AF, Wolpert DM (2002) Sources of signal-dependent noise during isometric force production. J Neurophysiol 88 (3):1533-1544.

31. Kurdak SS, Özgünen K, Adas Ü, Zeren C, Aslangiray B, Yazýcý Z, Korkmaz S (2005) Analysis of isokinetic knee extension/flexion in male elite adolescent wrestlers. J Sports Sci Med 4:489-498.

32. Lindeman RH, Merenda PF, Gold RZ (1980) Introduction to bivariate and multivariate analysis. Scott, Foresman, Glenview, IL.

33. McHugh MP, Tyler TF, Browne MG, Gleim GW, Nicholas SJ (2002) Electromyographic predictors of residual quadriceps muscle weakness after anterior cruciate ligament reconstruction. Am J Sports Med 30 (3):334-339.

34. McNair PJ, Wood GA (1993) Frequency analysis of the EMG from the quadriceps of anterior cruciate ligament deficient individuals. Electromyogr Clin Neurophysiol 33 (1):43-48. 
35. Newcombe RG (1998) Two-sided confidence intervals for the single proportion: comparison of seven methods. Stat Med 17 (8):857-872.

36. Palmieri-Smith RM, Thomas AC (2009) A neuromuscular mechanism of posttraumatic osteoarthritis associated with ACL injury. Exerc Sport Sci Rev 37 (3):147-153.

37. Pua YH, Byrant AL, Steele JR, Newton RU, Wrigley TV (2008) Isokinetic dynamometry in anterior cruciate ligament injury and reconstruction. Ann Acad Med Singapore 37 (4):330-340.

38. Ross MD, Langford B, Whelan PJ (2002) Test-retest reliability of 4 single-leg horizontal hop tests. J Strength Cond Res 16 (4):617-622.

39. Rudolph KS, Axe MJ, Buchanan TS, Scholz JP, Snyder-Mackler L (2001) Dynamic stability in the anterior cruciate ligament deficient knee. Knee Surg Sports Traumatol Arthrosc 9 (2):62-71.

40. Salonikidis K, Amiridis IG, Oxyzoglou N, de Villareal ES, Zafeiridis A, Kellis E (2009) Force variability during isometric wrist flexion in highly skilled and sedentary individuals. Eur J Appl Physiol 107 (6):715-722.

41. Seidler-Dobrin RD, He J, Stelmach GE (1998) Coactivation to reduce variability in the elderly. Motor Control 2 (4):314-330.

42. Singh NB, Arampatzis A, Duda G, Heller MO, Taylor WR (2010) Effect of fatigue on force fluctuations in knee extensors in young adults. Philos Trans A Math Phys Eng Sci 368 (1920):2783-2798.

43. Tsepis E, Giakas G, Vagenas G, Georgoulis A (2004) Frequency content asymmetry of the isokinetic curve between ACL deficient and healthy knee. J Biomech 37 (6):857-864.

44. Yanagawa T, Shelburne K, Serpas F, Pandy M (2002) Effect of hamstrings muscle action on stability of the ACL-deficient knee in isokinetic extension exercise. Clin Biomech (Bristol, Avon) 17 (9-10):705-712.

45. Yao W, Fuglevand RJ, Enoka RM (2000) Motor-unit synchronization increases EMG amplitude and decreases force steadiness of simulated contractions. J Neurophysiol 83 (1):441-452. 
TABLE 1: Clinical and functional characteristics of the study patients

\begin{tabular}{|c|c|}
\hline Characteristics & Values \\
\hline Age (yr) & $222430 \quad(26 \pm 5.8)$ \\
\hline Men, no. $(\%)$ & $73(84 \%)$ \\
\hline Body mass index $\left(\mathrm{kg} / \mathrm{m}^{2}\right)$ & $21.523 .026 .1 \quad(23.8 \pm 3.3)$ \\
\hline KT-2000 difference at 30lbs (mm) & $304068 \quad(48 \pm 28)$ \\
\hline Tegner activity level (pre-injury) & $7.07 .09 .0 \quad(7.5 \pm 1.2)$ \\
\hline Lower Extremity Functional Scale & $576367 \quad(61.1 \pm 9.4)$ \\
\hline \multicolumn{2}{|l|}{ ACLD side } \\
\hline Quadriceps st & $1.692 .092 .45 \quad(2.06 \pm 0.51)$ \\
\hline Quadriceps t & $2.152 .412 .91 \quad(2.53 \pm 0.65)$ \\
\hline Hamstrings strength $(\mathrm{Nm} / \mathrm{kg})$ & $1.011 .181 .36 \quad(1.19 \pm 0.28)$ \\
\hline Hamstrings torque steadiness $(\mathrm{Hz})$ & $\begin{array}{llll}1.912 .28 & 2.60 & (2.28 \pm 0.46)\end{array}$ \\
\hline Hop for distance $(\mathrm{cm})$ & $115138156 \quad(134 \pm 32)$ \\
\hline Six-metre hop for velocity $(\mathrm{m} / \mathrm{s})$ & $2.032 .422 .89 \quad(2.35 \pm 0.67)$ \\
\hline Pain with isokinetic quadriceps testing & $003 \quad(1.75 \pm 2.42)$ \\
\hline gs testing & $003 \quad(1.47 \pm 2.08)$ \\
\hline Pain with 1 & 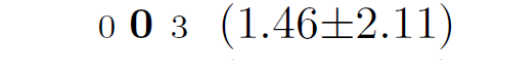 \\
\hline Pain with hop for velocity test & $003 \quad(1.66 \pm 2.27)$ \\
\hline \multicolumn{2}{|l|}{ Contralateral (healthy) side } \\
\hline Quadriceps strength $(\mathrm{Nm} / \mathrm{kg})$ & $2.48 \mathbf{2 . 7 6} 2.95 \quad(2.72 \pm 0.44)$ \\
\hline Quadriceps torque steadiness $(\mathrm{Hz})$ & $1.912 .122 .35 \quad(2.16 \pm 0.44)$ \\
\hline Hamstrings strength (Nm/kg) & $1.191 .391 .55 \quad(1.36 \pm 0.29)$ \\
\hline Hamstrings torque steadiness $(\mathrm{Hz})$ & 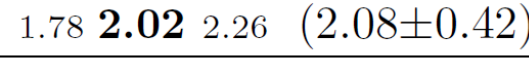 \\
\hline
\end{tabular}

$a b \quad c$ represent the lower quartile $a$, the median $b$, and the upper quartile $c$ for continuous variables. Values are mean $\pm \mathrm{SD}$ unless otherwise indicated 
TABLE 2. Multivariable association between isokinetic knee variables and hop distance

\begin{tabular}{lcccc}
\hline Variables & Comparison & Effect* & $95 \%$ CI & $P$-value \\
\hline Age (years) & 30 vs 22 & -1.6 & -8.5 to 5.3 & N.S. \\
Knee Pain & 1.43 vs -0.98 & -8.2 & -16.4 to 0.01 & N.S. \\
Sex & Female vs Male & -19.8 & -33.5 to -6.0 & $<0.01$ \\
Quadriceps Strength $(\mathrm{Nm} / \mathrm{kg})$ & 2.45 vs 1.69 & 23.5 & 15.2 to 31.7 & $<0.001$ \\
Quadriceps Steadiness $(\mathrm{Hz})$ & 1.46 vs 1.08 & -12.7 & -19.4 to -5.9 & $<0.001$ \\
\hline Variables & Comparison & Effect* & $95 \%$ CI & $P$-value \\
\hline Age (years) & 30 vs 22 & -0.64 & -7.8 to 9.1 & N.S. \\
Knee Pain & 1.43 vs -0.98 & -0.78 & -10.7 to 8.5 & N.S. \\
Sex & Female vs Male & -13.3 & -30.3 to 3.7 & N.S. \\
Hamstrings Strength $(\mathrm{Nm} / \mathrm{kg})$ & 1.36 vs 1.01 & 17.3 & 9.0 to 25.5 & $<0.001$ \\
Hamstrings Steadiness $(\mathrm{Hz})$ & 1.30 vs 0.96 & -6.8 & -16.0 to 2.5 & N.S. \\
\hline
\end{tabular}

* To facilitate the clinical interpretation of model results, effect represents the change in predicted single-legged hop distance $(\mathrm{cm})$ associated with increasing each continuous variable from the first quartile (Q1) to the third quartile (Q3). For example, an increase from $1.08 \mathrm{~Hz}$ (Q1) to $1.46 \mathrm{~Hz}$ (Q3) in quadriceps steadiness (torque frequency) was independently associated with a $12.7 \mathrm{~cm}$ decrease in hop distance.

TABLE 3. Multivariable association between isokinetic knee variables and hop velocity

\begin{tabular}{lcccc}
\hline Variables & Comparison & Effect* & $95 \%$ CI & $P$-value \\
\hline Age (years) & 30 vs 22 & -0.04 & -0.19 to 0.11 & N.S. \\
Knee Pain & 1.43 vs -0.98 & -0.16 & -0.33 to 0.02 & N.S. \\
Sex & Female vs Male & -0.20 & -0.51 to 0.09 & N.S. \\
Quadriceps Strength $(\mathrm{Nm} / \mathrm{kg})$ & 2.45 vs 1.69 & 0.54 & 0.36 to 0.71 & $<0.001$ \\
Quadriceps Steadiness $(\mathrm{Hz})$ & 1.46 vs 1.08 & -0.24 & -0.38 to -0.09 & $<0.01$ \\
\hline Variables & Comparison & Effect* & $95 \%$ CI & $P$-value \\
\hline Age (years) & 30 vs 22 & 0.02 & -0.15 to 0.20 & N.S. \\
Knee Pain & 1.43 vs -0.98 & -0.02 & -0.21 to 0.17 & N.S. \\
Sex & Female vs Male & -0.07 & -0.42 to 0.28 & N.S. \\
Hamstrings Strength $(\mathrm{Nm} / \mathrm{kg})$ & 1.36 vs 1.01 & 0.38 & 0.21 to 0.55 & $<0.001$ \\
Hamstrings Steadiness $(\mathrm{Hz})$ & 1.30 vs 0.96 & -0.22 & -0.41 to -0.03 & 0.02 \\
\hline
\end{tabular}

* To facilitate the clinical interpretation of model results, effect represents the change in predicted 6-metre hop velocity $(\mathrm{m} / \mathrm{s})$ associated with increasing each continuous variable from the first quartile (Q1) to the third quartile (Q3). For example, an increase from $1.08 \mathrm{~Hz}$ (Q1) to $1.46 \mathrm{~Hz}$ (Q3) in quadriceps steadiness (torque frequency) was independently associated with a $0.24 \mathrm{~m} / \mathrm{s}$ decrease in hop velocity. 


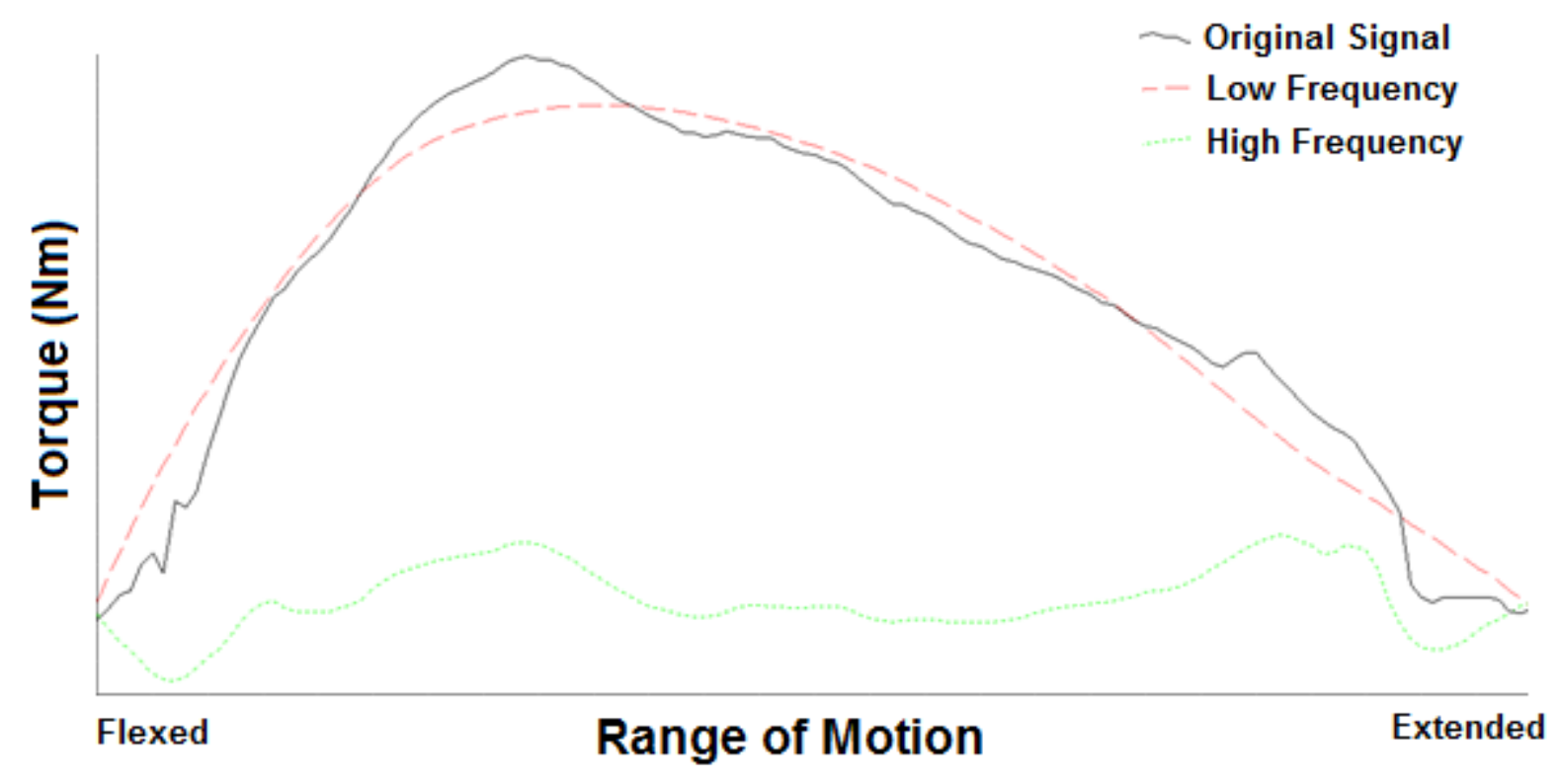

FIGURE 1 - Example data for a quadriceps test repetition with the original (raw), low frequency, and high frequency signal plotted. The mean instantaneous frequency (MIF) analysis performed in this study was only performed on the high frequency data.

Consequently, the MIF value represents the mean frequency of the fluctuations occurring at speeds greater than the underlying fundamental frequency of the movement, which is controlled due to the fixed velocity of the movement. 

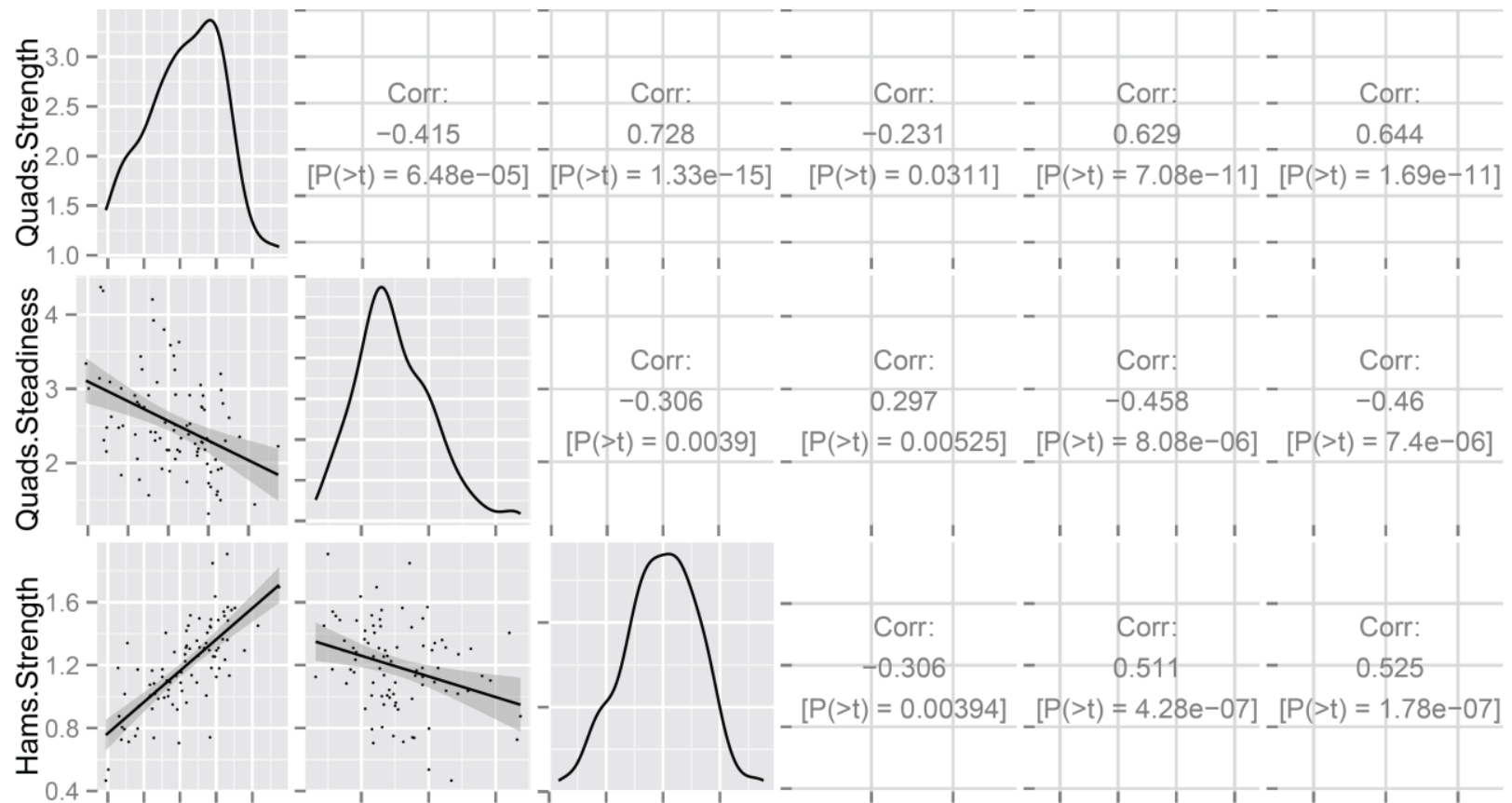

Corr:

0.297

Corr:

$-0.458$

Corr:

$-0.46$
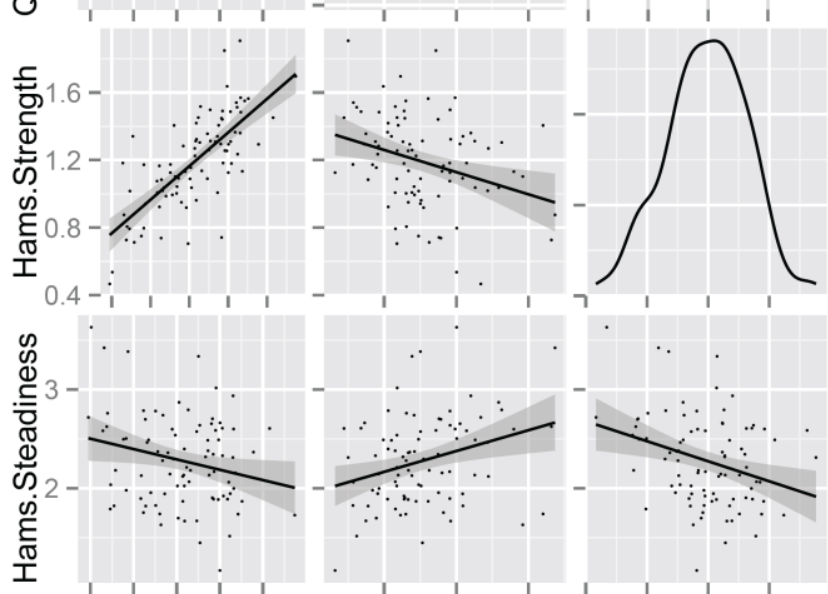

$[P(>t)=0.00525]$

$\left[P(>t)=8.08 e^{-06]}[P(>t)=7.4 e-06]\right.$
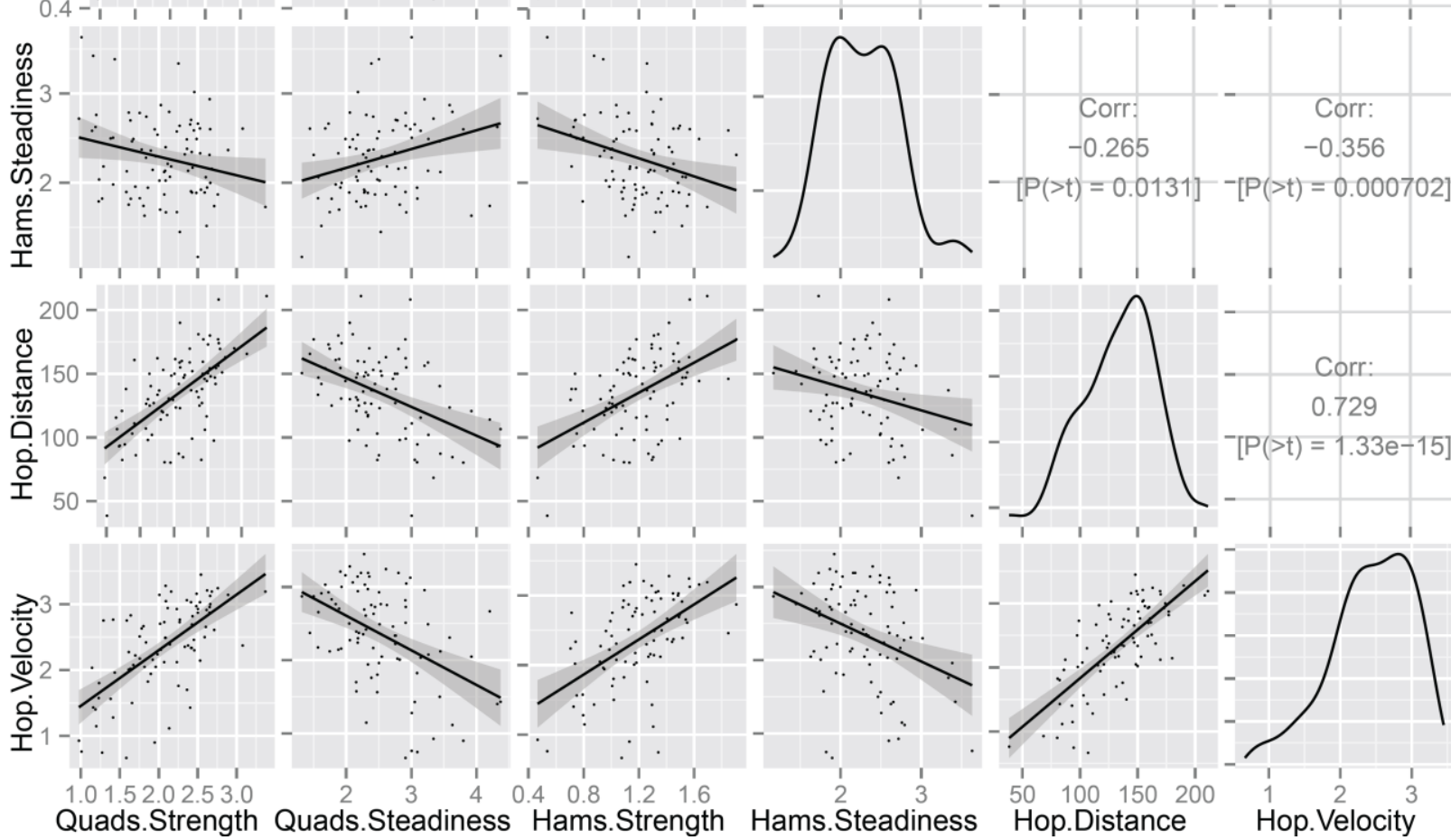

Quads.Steadiness Hams.Strength

ams.Steadiness

Hop.Distance

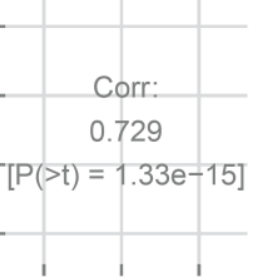

FIGURE 2 - Scatterplot matrix of isokinetic knee measures, hop distance, and 6-metre hop velocity. Diagonal panels show the kernel density plots of the variables. Lower diagonal panels show the scatterplots with loess smoothers and shaded regions are 95\% CIs for the regression estimates. Upper diagonal panels show the Pearson's coefficients with $P$-values. 


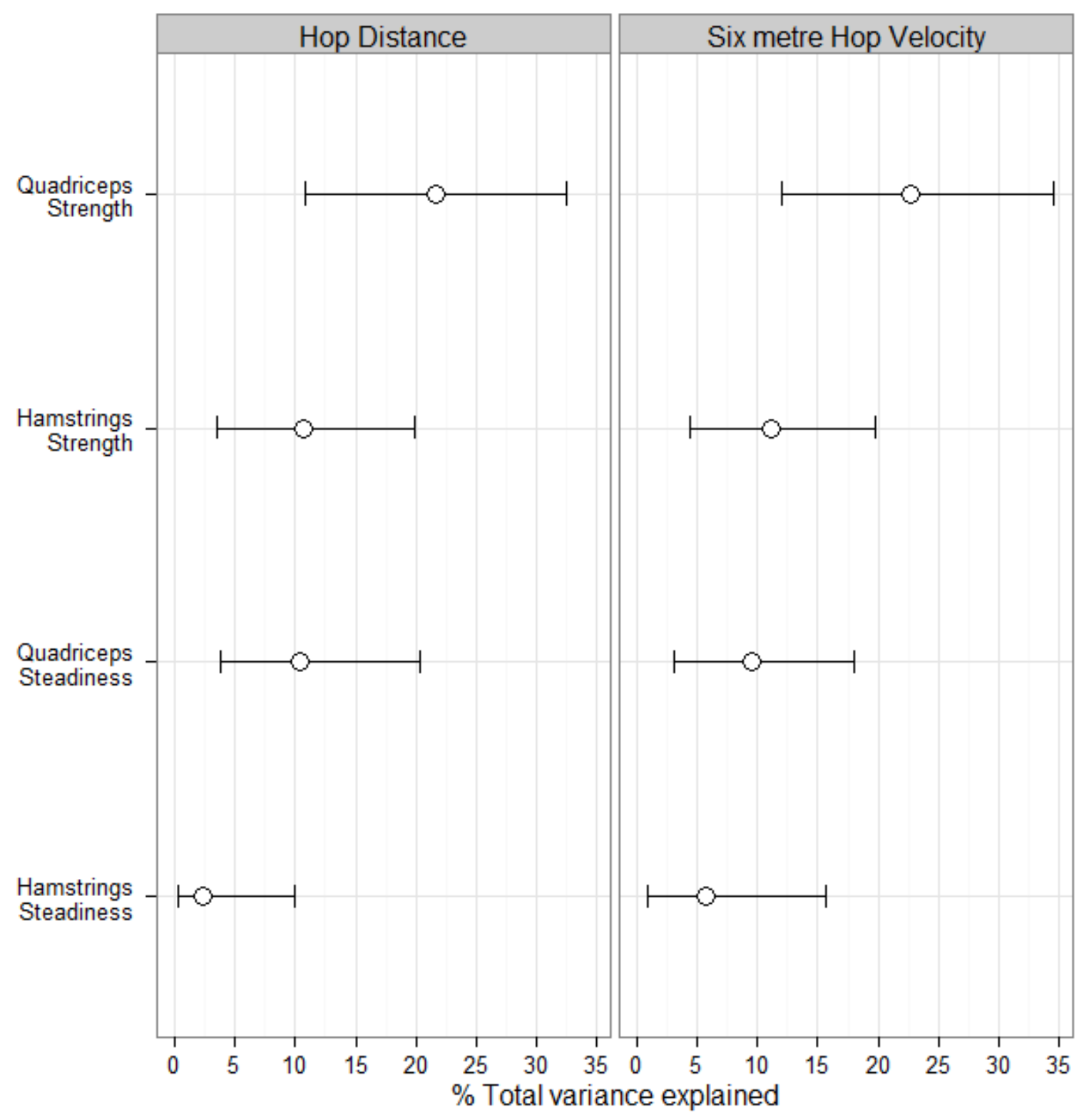

FIGURE 3 - Relative importance of isokinetic quadriceps and hamstrings variables for predicting hop distance (left panel) and 6-metre hop velocity (right panel) based on the Lindeman, Merenda, and Gold (LMG) metric. Error bars are 95\% bootstrap confidence intervals. 